\title{
Locally 3-arc-transitive regular covers of complete bipartite graphs
}

\author{
Eric Swartz \\ Department of Mathematics \\ College of William and Mary \\ P.O. Box 8795 \\ Williamsburg, VA 23187, U.S.A. \\ easwartz@wm. edu
}

Submitted: Jun 24, 2013; Accepted: Apr 15, 2016; Published: Apr 29, 2016

Mathematics Subject Classifications: 05C25, 05E18

\begin{abstract}
In this paper, locally 3 -arc-transitive regular covers of complete bipartite graphs are studied, and results are obtained that apply to arbitrary covering transformation groups. In particular, methods are obtained for classifying the locally 3-arctransitive graphs with a prescribed covering transformation group, and these results are applied to classify the locally 3 -arc-transitive regular covers of complete bipartite graphs with covering transformation group isomorphic to a cyclic group or an elementary abelian group of order $p^{2}$.
\end{abstract}

Keywords: Locally $s$-arc-transitive graphs; Graph automorphisms; Regular covers; Group actions

\section{Introduction}

We will assume that all graphs are finite, simple, undirected, and connected, unless otherwise stated. An $s$-arc of a graph $\Gamma$ is a sequence of vertices $\left(\alpha_{0}, \alpha_{1}, \cdots, \alpha_{s}\right)$ such that $\alpha_{i}$ is adjacent to $\alpha_{i+1}$ and $\alpha_{i-1} \neq \alpha_{i+1}$ for all $i, 1 \leqslant i \leqslant s-1$. Note that vertices can be repeated as long as $\alpha_{i-1} \neq \alpha_{i+1}$. An automorphism of the graph $\Gamma$ is a permutation of the vertices that preserves adjacency and nonadjacency. The set of all automorphisms of the graph $\Gamma$ forms a group and is denoted $\operatorname{Aut}(\Gamma)$. Given a subgroup $G \leqslant \operatorname{Aut}(\Gamma), \Gamma$ is $(G, s)$-arc-transitive if $\Gamma$ contains an $s$-arc and any $s$-arc in $\Gamma$ can be mapped to any other $s$-arc in $\Gamma$ via an element of $G$. The graph is locally $(G, s)$-arc-transitive if $\Gamma$ contains an $s$-arc and, for any vertex $\alpha$ of $\Gamma$, any $s$-arc starting at $\alpha$ can be mapped to any other $s$-arc starting at $\alpha$ via an element of $G$. In the cases that such a group $G$ exists, the graph $\Gamma$ is said to be $s$-arc-transitive or locally s-arc-transitive, respectively. Note that it is possible 
for a graph to be locally $(G, s)$-arc-transitive but for $G$ to be intransitive on the set of vertices. (As an example, one could take the complete bipartite graph $K_{2,3}$.) On the other hand, when $\Gamma$ is locally $(G, s)$-arc-transitive and every vertex in $\Gamma$ is adjacent to at least two other vertices, $G$ is transitive on the edges of $\Gamma$.

The study of $s$-arc-transitive graphs began with Tutte's seminal studies of cubic graphs $[27,28]$. Tutte proved that if $\Gamma$ is a cubic $(G, s)$-arc-transitive graph, then $s \leqslant 5$ (and that this upper bound is the best possible). Later, Weiss demonstrated that if the valency is at least three, then $s \leqslant 7$ [30]. Whereas Tutte's result used clever elementary methods, Weiss's result relied on the Classification of Finite Simple Groups (CFSG). On the other hand, locally $s$-arc-transitive graphs give geometric descriptions of rank 2 amalgams. The proof that $s \leqslant 9$ for any locally $s$-arc-transitive graph with all vertex valencies at least three [29] relies heavily on knowledge of amalgams and the classification of weak (B,N)pairs of rank 2 by Delgado and Stellmacher [2] and further demonstrates the deep link between the two concepts. Moreover, locally $s$-arc-transitive graphs arise as incidence graphs of highly symmetric structures $[19,31]$ and are important in their study. For instance, the only examples of Moufang generalized octagons are the Ree-Tits generalized octagons [26], the incidence graphs of which are locally 9-arc-transitive graphs.

A recent paper by Giudici, Li, and Praeger [11] set forth a program for the study of locally $s$-arc-transitive graphs. First, any locally $(G, s)$-arc-transitive graph that is $G$ vertex transitive is a $(G, s)$-arc-transitive graph, and many have been classified under a similar program initiated by Praeger in [23]. Second, since any locally $(G, s)$-arc-transitive graph of valency at least two is edge transitive, any locally $(G, s)$-arc-transitive graph that is vertex intransitive must be bipartite with vertex set $V \Gamma=\Delta_{1} \cup \Delta_{2}$ such that $G$ is transitive on each of $\Delta_{1}$ and $\Delta_{2}$, respectively. Third, there is a characterization of the graphs containing vertices of valency two [12, Theorem 6.2, Corollary 6.3]. Thus, the focus is on the graphs with minimum valency at least three that are $G$-vertex intransitive.

The breakthrough for studying these remaining $G$-vertex intransitive graphs is the normal quotient method from [11]. Let $\Gamma$ be a graph with a group of automorphisms $G$. If $G$ has a normal subgroup $N$ that acts intransitively on the vertices of $\Gamma$, define the (normal) quotient graph $\Gamma_{N}$ to have vertex set the $N$-orbits of vertices of $\Gamma$, and two $N$-orbits $\Sigma_{1}$ and $\Sigma_{2}$ are adjacent in $\Gamma_{N}$ if and only if there exist vertices $\alpha \in \Sigma_{1}$ and $\beta \in \Sigma_{2}$ such that $\alpha$ is adjacent to $\beta$ in $\Gamma$. The original graph $\Gamma$ is said to be a regular cover of $\Gamma_{N}$ if each $N$-orbit $\Sigma_{2}$ adjacent to $\Sigma_{1}$ contains exactly one vertex adjacent to $\alpha$ in $\Gamma$ for each $\alpha \in \Sigma_{1}$, and in this case $N$ is referred to as the covering transformation group. (See also Section 2 for different yet equivalent topological definitions.) Giudici, Li, and Praeger [11] showed that if $\Gamma$ is a locally $(G, s)$-arc-transitive graph, then $\Gamma_{N}$ is a locally $(G / N, s)$-arc-transitive graph. This insight led to the following framework for studying locally $s$-arc-transitive graphs:

(I) Understand the basic graphs, that is, those for which there is no nondegenerate normal quotient. A single vertex, a single edge, and a star, i.e., graphs of the form $K_{1, m}$ for some $m>1$ [11, Theorem 1.1], are the degenerate normal quotients in this context. 
(II) Understand the regular covers of the basic graphs.

The basic graphs will arise when the group $G$ acting on the graph has no normal subgroup with more than two orbits. With this in mind, a group $G$ acting transitively on a set $\Omega$ is said to be quasiprimitive if every nontrivial normal subgroup $N$ of $G$ is transitive on $\Omega$. Given the above definition of a quotient graph, it is clear that quasiprimitive groups are precisely the groups that give rise to basic graphs. The quasiprimitive groups were classified by Praeger [23] and refined into the following eight types [24]: holomorph of abelian group (HA), holomorph simple (HS), compound holomorph (HC), almost simple (AS), twisted wreath product (TW), simple diagonal (SD), compound diagonal (CD), and product action (PA). The main distinguishing factor among these different quasiprimitive actions is the socle of the quasiprimitive group $G$, which is the $\operatorname{subgroup} \operatorname{soc}(G)$ of $G$ generated by all minimal normal subgroups of $G$. There are four fundamentally different kinds of basic graphs for vertex intransitive locally $(G, s)$-arc-transitive graphs, where $s \geqslant 2$ :

[Complete Bipartite] Complete bipartite graphs $K_{m, n}$, where $m \neq n$;

[Star Normal Quotient] $G$ acts faithfully on both orbits of vertices $\Delta_{1}$ and $\Delta_{2}$, but only acts quasiprimitively on $\Delta_{1}$. In this case, the quasiprimitive action of $G$ on $\Delta_{1}$ must be one of HA, HS, AS, PA, or TW [11, Theorem 1.3];

[Same Type] $G$ acts faithfully and quasiprimitively on both orbits of vertices $\Delta_{1}$ and $\Delta_{2}$ with the same quasiprimitive type on each part, and the action of $G$ must be one of HA, TW, AS, or PA [11, Theorem 1.2];

[Different Type] $G$ acts faithfully and quasiprimitively on both orbits of vertices $\Delta_{1}$ and $\Delta_{2}$ with a different quasiprimitive action on each part. In this case, $G$ must act with type SD on $\Delta_{1}$ and with type PA on $\Delta_{2}$ [11, Theorem 1.2].

The graphs in [Star Normal Quotient] have a (degenerate) quotient that is isomorphic to the star $K_{1, m}$ for some $m>1$. This is the reason these graphs are collectively referred to as those with a star normal quotient.

While progress has been made in understanding the basic graphs $[9,10,11,12,13$, $19,25]$, there has been very little work done toward understanding the regular covers of locally $s$-arc-transitive graphs, which constitutes half of the program. The recent work $[1,8,20,21,22]$ on regular covers of finite symmetric graphs is limited by only considering a single graph at a time and restricting to abelian covering transformation groups. The study of regular covers of large families of symmetric graphs collectively has only been undertaken in a few specific cases $[6,7,16]$, even though studying regular covers of large classes of graphs is necessary to understanding symmetric graphs as a whole.

In order to introduce the main results of this paper, we need the following notation. Let $\alpha$ be a vertex of a graph $\Gamma$, and let $G \leqslant \operatorname{Aut}(\Gamma)$. We denote the vertices adjacent to $\alpha$ in $\Gamma$ by $\Gamma(\alpha)$, and the permutation group induced by $G_{\alpha}$ on $\Gamma(\alpha)$ will be denoted by 
$G_{\alpha}^{\Gamma(\alpha)}$. For any two vertices $\alpha, \beta$ of $\Gamma$, we define the distance function $d(\alpha, \beta)$ to be the length of the shortest path between $\alpha$ and $\beta$ in $\Gamma$. Given a natural number $i$, we define

$$
G_{\alpha}^{[i]}:=\left\{g \in G \mid \beta^{g}=\beta \text { for all } \beta \text { satisfying } d(\alpha, \beta) \leqslant i\right\} .
$$

Note that $G_{\alpha}^{[1]}$ is a normal subgroup of $G_{\alpha}$, and $G_{\alpha}^{\Gamma(\alpha)} \cong G_{\alpha} / G_{\alpha}^{[1]}$.

In this paper, we study the locally 3 -arc-transitive regular covers of complete bipartite graphs. In Section 2, we obtain results that apply to locally 3-arc-transitive regular covers of complete bipartite graphs with arbitrary covering transformation group, culminating in the following result:

Theorem 1. Let $\Gamma=K_{m, n}$ with biparts of vertices $\Delta_{1}$ and $\Delta_{2},\left|\Delta_{1}\right|=m \leqslant n=\left|\Delta_{2}\right|$, $\mathcal{P}$ : $\tilde{\Gamma} \rightarrow \Gamma$ be a regular covering projection such that $H:=\mathrm{CT}(\mathcal{P})$ and $G \leqslant \operatorname{Aut}(\Gamma)$ lifts to $\tilde{G}$ such that $\tilde{\Gamma}$ is locally $(\tilde{G}, 3)$-arc-transitive. For vertices $\alpha, \beta \in \Delta_{i^{\prime}}$, define further $K_{m, n}^{\alpha, \beta} \cong$ $K_{2, n}$ to be the subgraph induced on $\{\alpha, \beta\} \cup \Delta_{i}$ and $H_{\beta}$ to be the covering transformation group of $\mathcal{P}_{\alpha \beta}: \tilde{\Sigma} \rightarrow K_{m, n}^{\alpha, \beta}$, where $\tilde{\Sigma}$ is a connected component of $\mathcal{P}^{-1}\left(K_{m, n}^{\alpha, \beta}\right)$. Then one of the following must hold for each $\Delta_{i}$ :

(1) $\left|\Delta_{i}\right|=p^{f}$ for some prime $p$ and $f \in \mathbb{N}$, and $H_{\beta}$ is an elementary abelian group of order $p^{f}$, or

(2) There exists $X \leqslant \operatorname{Aut}\left(H_{\beta}\right)$ such that $X$ has a 2-transitive action on $\left|\Delta_{i}\right|$ points.

Moreover, in either case, if $v \in \Delta_{i}$, then $G_{\alpha \beta v}^{\Delta_{i}}$ is isomorphic to a subgroup of $\operatorname{Aut}\left(H_{\beta}\right)$.

One inference that we can make from Theorem 1 is that elementary abelian covering transformation groups are the most likely to yield locally 3-arc-transitive regular covers of complete bipartite graphs. We apply Theorem 1 to cyclic covering transformation groups and elementary abelian covering transformation groups in Sections 3 and 4, respectively, and obtain the following classification results:

Theorem 2. Let $\Gamma=K_{m, n}, m \leqslant n, \mathcal{P}: \tilde{\Gamma} \rightarrow \Gamma$ be a regular covering projection such that $H:=\mathrm{CT}(\mathcal{P}) \neq 1$ is a cyclic group and $G \leqslant \operatorname{Aut}(\Gamma)$ lifts to $\tilde{G}$ such that $\tilde{\Gamma}$ is locally $(\tilde{G}, 3)$-arc-transitive. Then one of the following holds:

(1) $m=n=2$ and $H \cong \mathbb{Z}_{N}$, where $N$ is any positive integer at least 2 ;

(2) $m=2, n=p$ for some odd prime $p$, and $H \cong \mathbb{Z}_{p}$;

(3) $m=n=p$ for some odd prime $p$ and $H \cong \mathbb{Z}_{p}$.

Moreover, in each case the cover is unique (up to isomorphism of covering projections).

Theorem 3. Let $\Gamma=K_{m, n}$ with biparts of vertices $\Delta_{1}$ and $\Delta_{2},\left|\Delta_{1}\right|=m \leqslant n=\left|\Delta_{2}\right|$, $\mathcal{P}: \tilde{\Gamma} \rightarrow \Gamma$ be a regular covering projection such that $H:=\mathrm{CT}(\mathcal{P}) \cong Z_{p}^{d}$, p prime and $d \geqslant 1$, is an elementary abelian group and $G \leqslant \operatorname{Aut}(\Gamma)$ lifts to $\tilde{G}$ such that $\tilde{\Gamma}$ is locally $(\tilde{G}, 3)$-arc-transitive. Then one of the following must hold for each $\Delta_{i}$ : 
(1) $\left|\Delta_{i}\right|=p^{f}$ for some $f \leqslant d$, or

(2) There exists $X \leqslant \mathrm{GL}_{d}(p)$ such that $X$ has a 2-transitive action on $\left|\Delta_{i}\right|$ points.

Moreover, in either case, if $v \in \Delta_{i}$, then $G_{\alpha \beta v}^{\Delta_{i}}$ is isomorphic to a subgroup of $\operatorname{GL}_{d}(p)$.

We apply this result to covering transformation groups isomorphic to $\mathbb{Z}_{p} \times \mathbb{Z}_{p}$ to obtain the following classification:

Theorem 4. Let $H \cong \mathbb{Z}_{p} \times \mathbb{Z}_{p}$, where $p$ is a prime. The only complete bipartite graphs with a locally 3-arc-transitive regular cover whose covering transformation group is isomorphic to a subgroup of $H$ are $K_{2,2}, K_{2,3}, K_{2, p}, K_{2, p^{2}}, K_{3, p}, K_{3, p^{2}}, K_{p, p}, K_{p, p^{2}}$, and $K_{p^{2}, p^{2}}$.

Corollary 5. Let $\Gamma=K_{m, n}, m \leqslant n, \mathcal{P}: \tilde{\Gamma} \rightarrow \Gamma$ be a regular covering projection such that $H:=\mathrm{CT}(\mathcal{P}) \cong \mathbb{Z}_{p} \times \mathbb{Z}_{p}$ is an elementary abelian group of order $p^{2}$ and $G \leqslant \operatorname{Aut}(\Gamma)$ lifts to $\tilde{G}$ such that $\tilde{\Gamma}$ is locally $(\tilde{G}, 3)$-arc-transitive. Then one of the following holds:

(1) $m=2$ and $n=3$;

(2) $m=2$ and $n=p^{2}$;

(3) $m=3$ and $n=p$;

(4) $m=3$ and $n=p^{2}$;

(5) $m=p$ and $n=p^{2}$;

(6) $m=n=p^{2}$.

Moreover, in each case the cover is unique (up to isomorphism of covering projections).

\section{Results applicable to general covering transformation groups}

A covering projection $\mathcal{P}: \tilde{\Gamma} \rightarrow \Gamma$ maps $V \tilde{\Gamma}$ onto $V \Gamma$, preserving adjacency, such that for any vertex $\tilde{\alpha} \in V \tilde{\Gamma}$, the set of neighbors of $\tilde{\alpha}$ is mapped bijectively onto the neighbors of $\mathcal{P}(\tilde{\alpha})$. For a vertex $\alpha$ of $\Gamma$, the set of vertices $\mathcal{P}^{-1}(\alpha)$ that are mapped onto $\alpha$ by $\mathcal{P}$ is called the fiber over the vertex $\alpha$. An automorphism $g \in \operatorname{Aut}(\Gamma)$ lifts to $\tilde{g} \in \operatorname{Aut}(\tilde{\Gamma})$ if the following diagram commutes:

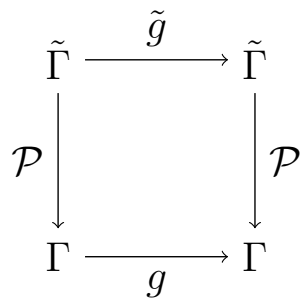


The lift of the trivial group (identity) is known as the group of covering transformations and is denoted $\mathrm{CT}(\mathcal{P}) . \tilde{\Gamma}$ is a regular cover of $\Gamma$ if $\mathrm{CT}(\mathcal{P})$ acts regularly on the set $\mathcal{P}^{-1}(\alpha)$ for all vertices $\alpha \in V \Gamma$. (Compare these to the equivalent definitions of Section 1.) A voltage assignment is a map $\xi: \vec{E} \Gamma \rightarrow H$, where $H$ is a group, such that $\xi(\alpha, \beta)=$ $\xi(\beta, \alpha)^{-1}$. For ease of notation, the voltage of the arc $(\alpha, \beta)$ will be denoted $\xi_{\alpha \beta}$. The derived covering graph $\tilde{\Gamma}$ of a voltage graph has vertex set $V \Gamma \times H$, where two vertices $\left(\alpha, h_{1}\right)$ and $\left(\beta, h_{2}\right)$ are adjacent iff $\alpha$ is adjacent to $\beta$ in $\Gamma$ and $h_{2}=\xi_{\alpha \beta} h_{1}$. The following theorem exhibits the deep connection between regular covers and derived covering graphs:

Theorem 6 ([15, Theorem 2.4.5, Section 2.5]). Every regular cover $\tilde{\Gamma}$ of a graph $\Gamma$ is a derived cover of a voltage graph. Moreover, if $\tilde{\Gamma}$ is connected we may assume that the edges of a (fixed but arbitrary) spanning tree of $\Gamma$ have been assigned the identity voltage.

Two covering projections $\mathcal{P}_{1}: \tilde{\Gamma}_{1} \rightarrow \Gamma_{1}$ and $\mathcal{P}_{2}: \tilde{\Gamma}_{2} \rightarrow \Gamma_{2}$ are said to be isomorphic if there exist graph isomorphisms $\sigma: \Gamma_{1} \rightarrow \Gamma_{2}$ and $\tilde{\sigma}: \tilde{\Gamma}_{1} \rightarrow \tilde{\Gamma_{2}}$ such that $\sigma \mathcal{P}_{1}=\mathcal{P}_{2} \tilde{\sigma}$. The following lemma connects isomorphisms of covers to isomorphisms of the covering transformation groups:

Lemma 7 ([21, Corollary 3.3]). Let $\mathcal{P}_{1}$ and $\mathcal{P}_{2}$ be two regular covers of a connected graph $\Gamma$, and let $H_{1}=\mathrm{CT}\left(\mathcal{P}_{1}\right)$ and $H_{2}=\mathrm{CT}\left(\mathcal{P}_{1}\right)$. The covering projections $\mathcal{P}_{1}$ and $\mathcal{P}_{2}$ are isomorphic if and only if there exists a group isomorphism $\tau: H_{1} \rightarrow H_{2}$ and $\sigma \in \operatorname{Aut}(\Gamma)$ such that $\tau \zeta_{1}=\zeta_{2} \sigma$, where, given a fixed vertex $\alpha$ of $\Gamma, \zeta_{1}$ and $\zeta_{2}$ are considered as epimorphisms $\zeta_{1}: \pi(\Gamma, \alpha) \rightarrow H_{1}$ and $\zeta_{2}: \pi\left(\Gamma, \alpha^{\sigma}\right) \rightarrow H_{2}$.

Throughout this section we will assume the following: $\Gamma$ is the complete bipartite graph $K_{m, n}$ with biparts $\Delta_{1}$ and $\Delta_{2}\left(\left|\Delta_{1}\right|=m,\left|\Delta_{2}\right|=n\right)$, $\Gamma$ has a regular cover $\tilde{\Gamma}$ with covering projection $\mathcal{P}: \tilde{\Gamma} \rightarrow \Gamma$, and the fiber-preserving automorphisms $\tilde{G}$ of $\tilde{\Gamma}$ act locally 3-arc-transitively on $\tilde{\Gamma}$. We will identify the vertices of $\Delta_{1}$ with lowercase Greek letters and vertices of $\Delta_{2}$ simply with the set $\{0,1,2, \cdots, n-1\}$. By Theorem $6, \tilde{\Gamma}$ is isomorphic to the derived graph of a voltage graph, where voltages are assigned to the co-tree edges with respect to some spanning tree $T$ of $\Gamma$. It does not matter which spanning tree we choose, and so we will choose the "double star" obtained by giving the identity voltage to all edges incident with either the vertex $\alpha \in \Delta_{1}$ or the vertex $0 \in \Delta_{2}$.

We will let $H=\mathrm{CT}(\mathcal{P})$ and assume that $H \neq 1$ to assure that we have a nontrivial cover. Moreover, we will let $\xi$ be the function from the edge set of $\Gamma$ to $H$ with $\xi_{i \beta}$ denoting the voltage assigned to the arc $(i, \beta)$. The following lemma gives explicit criteria for an automorphism of a graph to lift.

Lemma 8 ([21, Propositions 3.1, 5.1]). An automorphism g of $\Gamma$ lifts to an automorphism $\tilde{g}$ of $\tilde{\Gamma}$ if and only if there exists a group automorphism $g^{\# \alpha} \in \operatorname{Aut}(H)$ such that $\xi_{W}^{g^{\# \alpha}}=$ $\xi_{W^{g}}$, where $W$ runs over a generating set for all closed walks based at a single vertex $\alpha$. Moreover, if $H$ is abelian, the automorphism $g^{\# \alpha}$ does not depend on the choice of base vertex $\alpha$.

Note that by Giudici, Li, Praeger [11, Theorem 1.1], if $G=\tilde{G} / \mathrm{CT}(\mathcal{P})$, then $\Gamma$ is a locally $(G, 3)$-arc-transitive graph, and the group of automorphisms $G$ of $\Gamma$ lifts to $\tilde{\Gamma}$. 
At this point, we may associate each voltage $\xi_{i \gamma}$ with the closed walk $(\alpha, i, \gamma, 0, \alpha)$ based at $\alpha$. We note that for any fixed $\beta \neq \alpha \in \Delta_{1}$ and any $g \in G_{\alpha \beta}$, we may use Lemma 8 to define a homomorphism $\varphi_{\alpha \beta}: G_{\alpha \beta} \rightarrow \operatorname{Aut}(H)$ as follows: for any $g \in G_{\alpha \beta}$, we choose the lift $\tilde{g}$ of $g$ such that $(\alpha, 1)^{\tilde{g}}=\left(\alpha^{g}, 1\right)$. For a given $h \in H$, we may define the induced automorphism on $H$ by the action of $\tilde{g}$ on $H$ via the mapping $(\alpha, h)^{\tilde{g}}=\left(\alpha^{g}, h^{\varphi_{\alpha \beta}(g)}\right)$. Furthermore, we note that $\xi_{i \gamma}^{\varphi_{\alpha \beta}(g)}=\xi_{0^{g} \gamma^{g}}^{-1} \xi_{i^{g} \gamma^{g}}$, where $\xi_{0 \delta}=1$ for any $\delta \in \Delta_{1}$.

Lemma 9. For any vertex $\beta \neq \alpha \in \Delta_{1}, G_{\alpha \beta}^{\Delta_{2}}$ is 2-transitive. Moreover, the voltages assigned to the co-tree edges of $\Gamma$ must all have the same order, and, for any two distinct vertices $i, j \in \Delta_{2}, \xi_{i \beta} \neq \xi_{j \beta}$.

Proof. The fact that $G_{\alpha \beta}^{\Delta_{2}}$ is 2-transitive follows immediately from local 3-arc-transitivity. Note that any 3 -arc beginning at $\alpha$ and ending at 0 , say $(\alpha, i, \beta, 0)$, defines a unique 4-cycle $(\alpha, i, \beta, 0, \alpha)$. Since $\Gamma$ is locally $(G, 3)$-arc-transitive, for any $\gamma \in \Delta_{1}$ and $j \in \Delta_{2}$, there exists $g \in G$ such that $(\alpha, i, \beta, 0)^{g}=(\alpha, j, \gamma, 0)$. Hence the voltage of the first walk, $\xi_{i \beta}$, must be mapped to the voltage of the second walk, $\xi_{j \gamma}$, by the induced action of the element $g$ on $H$ (i.e., $\varphi_{\alpha \beta}(g) \in \operatorname{Aut}(H)$, as defined above). Thus the voltages on co-tree edges have the same order. Finally, if $\xi_{i \beta}=\xi_{j \beta}$ for some $i \neq j$, then the voltage of the closed walk $(\alpha, i, \beta, j, \alpha)$ is trivial. However, for any $0 \neq k \in \Delta_{2}$ and $\alpha \neq \gamma \in \Delta_{1}$, there is $g \in G_{\alpha}$ such that $(\alpha, i, \beta, j)^{g}=(\alpha, k, \gamma, 0)$, and so $\xi_{k \gamma}=1^{\varphi_{\alpha \beta}(g)}=1$, and all co-tree edges have trivial voltage.

Recall that the socle of a group $X$, denoted by $\operatorname{soc}(X)$ is defined to be the subgroup generated by all minimal normal subgroups of $X$. Since $G_{\alpha \beta}^{\Delta_{2}}$ is a 2-transitive group for any $\beta \neq \alpha \in \Delta_{1}$, by Burnside's Theorem (see [4, Theorem 4.1B], for instance), $\operatorname{soc}\left(G_{\alpha \beta}^{\Delta_{2}}\right)$ is either an elementary abelian $p$-group that acts regularly on $\Delta_{2}$ for some prime $p$ or a nonabelian simple group that acts nonregularly on $\Delta_{2}$. Moreover, in each case the socle is the unique minimal normal subgroup. Henceforth we will assume that $\beta \neq \alpha \in \Delta_{1}$ is fixed and let $S:=\operatorname{soc}\left(G_{\alpha \beta}^{\Delta_{2}}\right)$.

We say that a group $X$ is involved in the group $Y$ if there exist subgroups $Y_{1} \triangleleft Y_{2} \leqslant Y$ such that $Y_{2} / Y_{1} \cong X$. Let $\phi: G_{\alpha \beta} \rightarrow G_{\alpha \beta}^{\Delta_{2}}$ be the natural projection with $\operatorname{Ker}(\phi)=G_{\alpha \beta}^{[1]}$. Since $G_{\alpha \beta}^{\Delta_{2}}$ has a unique minimal normal subgroup $S$, by the Correspondence Theorem there exists a subgroup $T \triangleleft G_{\alpha \beta}$ such that $G_{\alpha \beta}^{[1]} \leqslant T$ and $T / G_{\alpha \beta}^{[1]} \cong S$. Note that, since $S$ is a minimal normal subgroup of $G_{\alpha \beta}^{\Delta_{2}}$, if $N<T$ and $N$ is normal in $G_{\alpha \beta}$, then $N \leqslant G_{\alpha \beta}^{[1]}$.

Proposition 10. Let $\mathcal{P}: \tilde{\Gamma} \rightarrow \Gamma$ be a covering projection with covering transformation group $H$. If $\tilde{G}$ is the lift of the group $G$, the cover $\tilde{\Gamma}$ is locally $(\tilde{G}, 3)$-arc-transitive, and $S:=\operatorname{soc}\left(G_{\alpha \beta}^{\Delta_{2}}\right)$ is a nonabelian finite simple group, then $S$ is involved in $\operatorname{Aut}(H)$.

Proof. Let $\left|\Delta_{2}\right|=n$. First, we note that the group $G_{\alpha \beta 0}$, the stabilizer of the vertex $0 \in \Delta_{2}$ in the group $G_{\alpha \beta}$, transitively permutes the vertices $1,2, \ldots, n-1$. Since $\xi_{i \beta}$ is the voltage of the cycle $(\alpha, i, \beta, 0, \alpha)$, for any $g \in G_{\alpha \beta 0}$, we have $\xi_{i \beta}^{g^{\varphi_{\alpha \beta}(g)}}=\xi_{i^{g} \beta}$. By Lemma 9, $\xi_{i \beta}=\xi_{j \beta}$ only if $i=j$. Hence, for any such $g \in G_{\alpha \beta 0}$, if it induces a trivial action on $H$, then $i^{g}=i$ for all $i, 1 \leqslant i \leqslant n-1$, which implies that $g \in G_{\alpha \beta}^{[1]}$. 
As noted above, there is $T \leqslant G_{\alpha \beta}$ such that $G_{\alpha \beta}^{[1]} \leqslant T$ and $T / G_{\alpha \beta}^{[1]} \cong S$. If $S$ is not involved in $\operatorname{Aut}(H)$, then $T \leqslant \operatorname{Ker}\left(\varphi_{\alpha \beta}\right)$. However, by the Classification of Finite Simple Groups [14], the induced action of $S_{0}$ on $\{1, \ldots, n-1\}$ is nontrivial. Hence $T_{0}$ acts nontrivially on $\{1, \ldots, n-1\}$ and $T$ is not contained in $\operatorname{Ker}\left(\varphi_{\alpha \beta}\right)$. This means that $\operatorname{Ker}\left(\varphi_{\alpha \beta}\right) \cap T \leqslant G_{\alpha \beta}^{[1]}$. Therefore, $\varphi_{\alpha \beta}(T) / \varphi_{\alpha \beta}\left(G_{\alpha \beta}^{[1]}\right) \cong S$, and $S$ is involved in $\operatorname{Aut}(H)$.

Proposition 11. Let $\mathcal{P}: \tilde{\Gamma} \rightarrow \Gamma$ be a covering projection with covering transformation group $H$. If $\tilde{G}$ is the lift of the group $G$, the cover $\tilde{\Gamma}$ is locally $(\tilde{G}, 3)$-arc-transitive, and $S:=\operatorname{soc}\left(G_{\alpha \beta}^{\Delta_{2}}\right)$ is an elementary abelian p-group for some prime $p$, then either $S$ is involved in $\operatorname{Aut}(H)$ or each voltage assigned to a co-tree edge of $\Gamma$ has order $p$.

Proof. Assume that $S$ is not involved in $\operatorname{Aut}(H)$. As noted above, there is $T \leqslant G_{\alpha \beta}$ such that $G_{\alpha \beta}^{[1]} \leqslant T$ and $T / G_{\alpha \beta}^{[1]} \cong S$. By the same argument as in the proof of Proposition 10 , if $g \in G_{\alpha \beta 0}$ and $\varphi_{\alpha \beta}(g)=1$, then $g \in G_{\alpha \beta}^{[1]}$. Hence, if $S$ is not involved in $\operatorname{Aut}(H)$, $T \leqslant \operatorname{Ker}\left(\varphi_{\alpha \beta}\right)$, and $T_{0}=T \cap G_{\alpha \beta 0} \leqslant G_{\alpha \beta}^{[1]}$.

We note that $T$ is transitive on $\Delta_{2}$, so there is $s \in T$ such that $0^{s}=1$. Recalling the epimorphism $\phi: G_{\alpha \beta} \rightarrow G_{\alpha \beta}^{\Delta_{2}}, \phi(s) \in S$ and has order $p$. Thus $s^{p} \in \operatorname{Ker}(\phi)=G_{\alpha \beta}^{[1]}$, and $\phi\left(s^{m}\right)=\phi\left(s^{n}\right)$ for $1 \leqslant m, n \leqslant p-1$ implies $m=n$. This means that there exist vertices $a_{2}, \ldots, a_{p-1} \in \Delta_{2}$ such that $1^{s}=a_{2}, a_{m}^{s}=a_{m+1}$ for $2 \leqslant m \leqslant p-2$, and $a_{p-1}^{s}=0$. On the one hand, $(\alpha, 1, \beta, 0, \alpha)^{s}=\left(\alpha, a_{2}, \beta, 1, \alpha\right)$, and so we have an induced action $\xi_{1 \beta}^{\varphi_{\alpha \beta}(s)}=\xi_{1 \beta}^{-1} \xi_{a_{2} \beta}$. On the other hand, we are assuming that $T \leqslant \operatorname{Ker}\left(\varphi_{\alpha \beta}\right)$, and so $\xi_{i \beta}^{\varphi_{\alpha \beta}(s)}=\xi_{i \beta}$. This implies that $\xi_{1 \beta}=\xi_{1 \beta}^{\varphi_{\alpha \beta}(s)}=\xi_{1 \beta}^{-1} \xi_{a_{2} \beta}$, and so $\xi_{a_{2} \beta}=\xi_{1 \beta}^{2}$. Proceeding similarly, by induction we see that $\xi_{a_{m} \beta}=\xi_{1 \beta}^{m}$ for $1 \leqslant m \leqslant p-1$.

Moreover, $G_{\alpha \beta}$ is 2-transitive on $\Delta_{2}$, so there exists $h \in G_{\alpha \beta}$ such that $0^{h}=1$ and $1^{h}=0$, which implies that $h s^{-1}=r \in G_{\alpha \beta 0}$. Noting that $0^{r}=0$ and $1^{r}=a_{p-1}$, we have an induced action $\xi_{1 \beta}^{\varphi_{\alpha \beta}(h)}=\xi_{1 \beta}^{-1}$. On the other hand,

$$
\xi_{1 \beta}^{\varphi_{\alpha \beta}(h)}=\left(\xi_{1 \beta}^{\varphi_{\alpha \beta}(r)}\right)^{\varphi_{\alpha \beta}(s)}=\xi_{a_{p-1} \beta}^{\varphi_{\alpha \beta}(s)}=\xi_{a_{p-1} \beta} .
$$

Hence $\xi_{1 \beta}^{-1}=\xi_{a_{p-1} \beta}=\xi_{1 \beta}^{p-1}$ and $\xi_{1 \beta}^{p}=1$. Since $H \neq 1,\left|\xi_{1 \beta}\right|=p$, and by Lemma 9 the order of every voltage assigned to a co-tree edge of $\Gamma$ has order $p$.

Proposition 12. If the group $T$ has a trivial induced action on $H$, where $\phi(T)=S=$ $\operatorname{soc}\left(G_{\alpha \beta}^{\Delta_{2}}\right)$ and $G_{\alpha \beta}^{[1]} \leqslant T$, then each element of an entire elementary abelian group of order $p^{f}$, where $p^{f}$ is the size of the bipart $\Delta_{2}$, must be assigned as voltages to the edges incident with the vertex $\beta \neq \alpha \in \Delta_{1}$.

Proof. By Propositions 10 and 11, if the lift of $T$ acts trivially on $H$, then $G_{\alpha \beta}^{\Delta_{2}}$ is an elementary abelian $p$-group that acts regularly on $\Delta_{2}$ for some prime $p$. Thus $\left|\Delta_{2}\right|=p^{f}$ for some $f \in \mathbb{N}$, and by Proposition 11, the order of each voltage assigned to a co-tree edge is $p$. For any $i \in \Delta_{2} \backslash\{0\}$, there exists $s_{i} \in T$ such that $0^{s_{i}}=i$, and, proceeding as in the proof of Theorem 11, $\xi_{i \beta}^{m}=\xi_{i} s_{i}^{m}$, and so each power of the voltage $\xi_{i \beta}$ is assigned to an edge incident with $\beta$. 
We will next show that $\xi_{i \beta} \xi_{j \beta}$ is the voltage assigned to some edge incident with $\beta$, where $i, j$ are distinct vertices in $\Delta_{2} \backslash\{0\}$. In particular, $\xi_{i \beta}^{-1}=\xi_{i \beta}^{p-1}=\xi_{\ell \beta}$ for some $\ell \in \Delta_{2}$. Since $G_{\alpha \beta}$ is 2-transitive on $\Delta_{2}$, there is $g \in G_{\alpha \beta}$ such that $(\alpha, i, \beta, 0, \alpha)^{g}=(\alpha, j, \beta, \ell, \alpha)$, and so $\xi_{i \beta}^{\varphi_{\alpha \beta}(g)}=\xi_{\ell \beta}^{-1} \xi_{j \beta}=\xi_{i \beta} \xi_{j \beta}$. There is also an element $s \in T$ such that $0^{s}=\ell$, and so $g s^{-1} \in G_{\alpha \beta 0}$. Hence

$$
\xi_{i \beta} \xi_{j \beta}=\xi_{i \beta}^{\varphi_{\alpha \beta}(g)}=\left(\xi_{i \beta}^{\varphi_{\alpha \beta}\left(g s^{-1}\right)}\right)^{\varphi_{\alpha \beta}(s)}=\xi_{i^{g s}-1}^{\varphi_{\alpha \beta}(s)}=\xi_{i^{g s}-1} \beta
$$

since by assumption $T$ has a trivial induced action on $H$. Hence all the voltages incident with $\beta$ are all distinct, have order $p$ (other than $\xi_{0 \beta}$, which is assigned the trivial voltage), and form a subgroup.

Finally, define $H_{\beta}$ to be the group of voltages that are assigned to edges incident with $\beta$. Note that the induced action of $G_{\alpha \beta 0}$ is transitive on $\Delta_{2} \backslash\{0\}$, and hence for any $i \neq j \in \Delta_{2} \backslash\{0\}$, there is $g \in G_{\alpha \beta 0}$ such that $i^{g}=j$. This implies that $\xi_{i \beta}^{\varphi_{\alpha \beta}(g)}=\xi_{j \beta}$, and so the induced action of $G_{\alpha \beta 0}$ on $H_{\beta} \backslash\{1\}$ is transitive. On the other hand, $H_{\beta}$ is a $p$-group, and so for some $z \in \Delta_{2} \backslash\{0\}, \xi_{z \beta} \in Z\left(H_{\beta}\right)$. The center is a characteristic subgroup, and therefore $H_{\beta}$ is elementary abelian.

Proposition 13. The stabilizer of a point in $G_{\alpha \beta}^{\Delta_{2}}$ is involved in $\operatorname{Aut}(H)$.

Proof. Suppose $g \in G_{\alpha \beta 0}$ and $\varphi_{\alpha \beta}(g)=1$. In particular, for all $i \in \Delta_{2} \backslash\{0\}, \xi_{i \beta}=$ $\xi_{i \beta}^{\varphi_{\alpha \beta}(g)}=\xi_{i^{g} \beta}$. By Lemma 9, this implies that $i^{g}=i$ for all $i \in \Delta_{2}$, and so $g \in G_{\alpha \beta}^{[1]}$. Hence $\operatorname{Ker}\left(\varphi_{\alpha \beta}\right) \leqslant G_{\alpha \beta}^{[1]}$, and so

$$
G_{\alpha \beta 0}^{\Delta_{2}} \cong \varphi_{\alpha \beta}\left(G_{\alpha \beta 0}\right) / \varphi_{\alpha \beta}\left(G_{\alpha \beta}^{[1]}\right)
$$

is involved in $\operatorname{Aut}(H)$.

Corollary 14. If the group $T$ has a nontrivial induced action on $H$, then $G_{\alpha \beta}^{\Delta_{2}}$ is involved in $\operatorname{Aut}(H)$.

Proof. Let $S=\operatorname{soc}\left(G_{\alpha \beta}^{\Delta_{2}}\right)$ and $K=G_{\alpha \beta 0}^{\Delta_{2}}$. Since $S$ is transitive on $\Delta_{2}$ and $K$ is the stabilizer of a point, $G_{\alpha \beta}^{\Delta_{2}}=S K$, and hence, by Propositions 10, 11, and 13,

$$
G_{\alpha \beta}^{\Delta_{2}}=S K \cong\left(\varphi_{\alpha \beta}(T) / \varphi_{\alpha \beta}\left(G_{\alpha \beta}^{[1]}\right)\right)\left(\varphi_{\alpha \beta}\left(G_{\alpha \beta 0}\right) / \varphi_{\alpha \beta}\left(G_{\alpha \beta}^{[1]}\right)\right) \cong \varphi_{\alpha \beta}\left(G_{\alpha \beta}\right) / \varphi_{\alpha \beta}\left(G_{\alpha \beta}^{[1]}\right) .
$$

We will next show that there exists a locally 3-arc-transitive regular cover of $K_{m, n}$ only if there exist locally 3-arc-transitive regular covers of $K_{2, m}$ and $K_{2, n}$, respectively. For a complete bipartite graph $K_{m, n}$ with $\left|\Delta_{1}\right|=m$ and $\left|\Delta_{2}\right|=n$, if $\gamma, \delta \in \Delta_{1}$, then we define $K_{m, n}^{\gamma, \delta} \cong K_{2, n}$ to be the subgraph induced on $\{\gamma, \delta\} \cup \Delta_{2}$. The following proof, which simultaneously simplifies and strengthens results from a previous version of this paper, comes from an anonymous referee:

Proposition 15. Let $\mathcal{P}: \tilde{\Gamma} \rightarrow K_{m, n}$ be a regular covering projection, and assume that $\tilde{\Gamma}$ is locally $(\tilde{G}, 3)$-arc-transitive, where $\tilde{G}$ is the lift of a group $G \leqslant \operatorname{Aut}\left(K_{m, n}\right)$. If $\tilde{\alpha} \in \mathcal{P}^{-1}(\alpha)$ and $\tilde{\beta} \in \mathcal{P}^{-1}(\beta)$ are vertices in the same connected component $\tilde{\Sigma}$ of $\mathcal{P}^{-1}\left(K_{m, n}^{\alpha, \beta}\right)$, then $\tilde{\Sigma}$ is locally $\left(\tilde{G}_{\{\tilde{\alpha}, \tilde{\beta}\}}, 3\right)$-arc-transitive. 
Proof. It is clear that the graphs $K_{m, n}^{\gamma, \delta} \cong K_{2, n}$ are isomorphic for each choice of $\gamma, \delta \in \Delta_{1}$. Moreover, given a vertex $0 \in \Delta_{2}$, the group $G_{0}$ is 2 -transitive on $\Delta_{1}$, and so for any $\gamma, \delta \in \Delta_{1}$, there is a subgroup of $G$ that takes $K_{m, n}^{\alpha, \beta}$ to $K_{m, n}^{\gamma, \delta}$. Since this group lifts by assumption, there is an isomorphism $\kappa: \mathcal{P}^{-1}\left(K_{m, n}^{\alpha, \beta}\right) \rightarrow \mathcal{P}^{-1}\left(K_{m, n}^{\gamma, \delta}\right)$ by Lemma 7 . Furthermore, the subgroup of $G$ for which $K_{m, n}^{\alpha, \beta}$ is invariant, namely $G_{\{\alpha, \beta\}}$, is a locally 3arc-transitive subgroup of $\operatorname{Aut}\left(K_{m, n}^{\alpha, \beta}\right)$. Since $G_{\{\alpha, \beta\}}$ lifts, by [5, Theorem 3], any connected component of $\mathcal{P}^{-1}\left(K_{m, n}^{\alpha, \beta}\right)$ is also locally $(\tilde{G}, 3)$-arc-transitive. The result follows.

Proposition 15 implies that, for a complete bipartite graph $K_{m, n}$ with a locally 3-arctransitive regular cover, if $\left|\Delta_{1}\right|=m,\left|\Delta_{2}\right|=n, \beta \in \Delta_{1}$, and $H_{\beta}$ is the group generated by the voltages assigned to the edges incident with $\beta$, then there exists a locally 3 arc-transitive regular cover of $K_{2, n}$ with covering transformation group isomorphic to $H_{\beta}$. Moreover, the voltage assigned to the $\operatorname{arc}(i, \beta)$ in $K_{2, n}$ is the same as that which is assigned to the $\operatorname{arc}(i, \beta)$ in $K_{m, n}^{\alpha, \beta}$, i.e., there is an isomorphism of covering projections. Note further that by Lemma 7 , for any vertices $\beta, \gamma \in \Delta_{1} \backslash\{\alpha\}$, there is a group isomorphism $\tau: H_{\gamma} \rightarrow H_{\beta}$, and the group $H_{\beta}$ is independent of the choice of $\beta$. We can now prove Theorem 1.

Proof of Theorem 1. By Proposition 15 , if $\left|\Delta_{i}\right|=n$, then $K_{2, n}$ has a locally $(\tilde{K}, 3)$-arctransitive regular cover with covering transformation group $H_{\beta}$, where $\tilde{K}$ is a lift of the group $K \leqslant \operatorname{Aut}\left(K_{2, n}\right)$. Let $\Delta$ be the bipart of size $n$ in $K_{2, n}$. Note that $K_{\alpha \beta}=K_{\alpha \beta}^{\Delta}$, so $K_{\alpha \beta}^{[1]}=1$. Let $T=\operatorname{soc}\left(K_{\alpha \beta}\right)$ and $\varphi_{\alpha \beta}: K_{\alpha \beta} \rightarrow \operatorname{Aut}\left(H_{\beta}\right)$ be the induced action, as above. If $\varphi_{\alpha \beta}(T)=1$, then, by Proposition $12, n=p^{f}$ for some prime $p$ and $f \in \mathbb{N}$, and $H_{\beta}$ is an elementary abelian group of order $p^{f}$. Otherwise, $T$ has a nontrivial induced action on $H_{\beta}$, and, by Corollary $14, K_{\alpha \beta}=K_{\alpha \beta}^{\Delta}$ is involved in $\operatorname{Aut}\left(H_{\beta}\right)$. Moreover, since $K_{\alpha \beta}^{[1]}=1$, we have $\varphi_{\alpha \beta}\left(K_{\alpha \beta}^{[1]}\right)=1$, and

$$
K_{\alpha \beta} \cong \varphi_{\alpha \beta}\left(K_{\alpha \beta}\right) / \varphi_{\alpha \beta}\left(K_{\alpha \beta}^{[1]}\right) \cong \varphi_{\alpha \beta}\left(K_{\alpha \beta}\right) .
$$

In this case, $K_{\alpha \beta}$, which has a 2-transitive action on $n=|\Delta|=\left|\Delta_{i}\right|$ elements, is isomorphic to a subgroup of Aut $\left(H_{\beta}\right)$. Using Proposition 13, a similar argument shows that, in either case, $K_{\alpha \beta v}^{\Delta_{i}} \cong G_{\alpha \beta v}^{\Delta_{i}}$ is isomorphic to a subgroup of $\operatorname{Aut}\left(H_{\beta}\right)$ for any $v \in \Delta_{i}$. The result follows.

\section{Locally 3-arc-transitive regular covers of complete bipartite graphs with cyclic covering transformation group}

Let $\Gamma=K_{m, n}$ have biparts $\Delta_{1}$ and $\Delta_{2}$, and assume that $\Gamma$ has a locally 3 -arc-transitive regular cover $\tilde{\Gamma}$ with covering projection $\mathcal{P}: \tilde{\Gamma} \rightarrow \Gamma$. We will also assume that the group of covering transformations is cyclic, i.e., let $H=\mathrm{CT}(\mathcal{P})=\langle\xi\rangle \cong \mathbb{Z}_{N}$ for some integer $N \in \mathbb{N}, N>1$, where the cyclic group will be viewed additively. As in the previous section, we fix $\beta \neq \alpha \in \Delta_{1}$, let $G$ be the group of automorphisms that lift, and let $T=T_{\alpha \beta}$ be the subgroup of $G_{\alpha \beta}$ that contains $G_{\alpha \beta}^{[1]}$ and $T / G_{\alpha \beta}^{[1]} \cong \operatorname{soc}\left(G_{\alpha \beta}^{\Delta_{2}}\right)$. Furthermore, $G_{\alpha \beta}$ has an induced action on $H$ given by $\varphi_{\alpha \beta}: G_{\alpha \beta} \rightarrow \operatorname{Aut}(H)$. 
Lemma 16. If $\left|\Delta_{2}\right|>2$, then the induced action of the group $T$ on $H$ is trivial, i.e., $\varphi_{\alpha \beta}(T)=1$.

Proof. By Theorem 1, if the induced action of $T$ on $H$ is not trivial, then there exists $X \leqslant \operatorname{Aut}\left(H_{\beta}\right)$ with a 2 -transitive action on $\left|\Delta_{2}\right|$ elements. On the other hand, $H$ is cyclic and generated by the voltages assigned to the edges of $\Gamma$, and so $H_{\beta} \leqslant H$ is also cyclic. (Actually, by Lemma 9, the voltages assigned to the edges of $\Gamma$ all have the same order, so $H_{\beta}=H$.) Thus $\operatorname{Aut}\left(H_{\beta}\right)$ is isomorphic to the group of units of $H_{\beta}$, which is abelian. When $\left|\Delta_{i}\right|>2$, no 2-transitive group on $\Delta_{i}$ is abelian, and so no such group $X$ can exist, a contradiction.

Lemma 17. If $\left|\Delta_{2}\right|>2$, then $G_{\alpha \beta}^{\Delta_{2}} \cong \mathbb{Z}_{p}: \mathbb{Z}_{p-1}$ and $H \cong \mathbb{Z}_{p}$.

Proof. By Lemma 16, the group $T$ has trivial induced action on $H$, i.e., $T \leqslant \operatorname{Ker}\left(\varphi_{\alpha \beta}\right)$. By Theorem 1, the group of covering transformations $H$ must be isomorphic to $\mathbb{Z}_{p}^{f}$, where $p$ is a prime and $f \in \mathbb{N}$. Since $H$ is cyclic, $H \cong \mathbb{Z}_{p}$ and $\left|\Delta_{2}\right|=p$. Moreover, by Proposition $12, \operatorname{soc}\left(G_{\alpha \beta}^{\Delta_{2}}\right) \cong \mathbb{Z}_{p}$, and hence $G_{\alpha \beta}^{\Delta_{2}} \cong \mathbb{Z}_{p}: \mathbb{Z}_{p-1}$.

Proposition 18. The only possibilities for $\Gamma=K_{m, n}$ are $K_{2, p}$ and $K_{p, p}$, where $p$ is a prime.

Proof. By Lemma 17, we may assume that $\left|\Delta_{2}\right|=n=p$ for some prime $p$. On the other hand, using the same reasoning, $\left|\Delta_{1}\right|=p^{\prime}$ for some prime $p^{\prime}$. If both $p, p^{\prime}>2$, then by Lemma 17 it is impossible for all edges to have voltages from both $\mathbb{Z}_{p}$ and $\mathbb{Z}_{p^{\prime}}$ unless $p=p^{\prime}$. Hence the only possibilities are $K_{2, p}$ and $K_{p, p}$, where $p$ is a prime.

Proof of Theorem 2. First, we note that Proposition 18 implies that $K_{2,2}, K_{2, p}$, and $K_{p, p}$ are the only three possibilities; all that remains is to show that unique covers actually exist in each listed case.

We note that $K_{2,2}$ is a four cycle, and the cover of $K_{2,2}$ derived from the group $\mathbb{Z}_{N}$ is a $4 N$-cycle. Moreover, for any two covering transformation groups $H_{1} \cong H_{2} \cong \mathbb{Z}_{N}$, there is an obvious isomorphism, and so by Lemma 7, there is a unique such cover up to isomorphism of covering projections.

Suppose now that $\left|\Delta_{1}\right|=m=2$ and $\left|\Delta_{2}\right|=n=p$, where $p$ is an odd prime. By Lemma 17, $N=p$. Continuing with the notation above, let $\Delta_{1}=\{\alpha, \beta\}$ and $\Delta_{2}=$ $\{0,1, \ldots, p-1\}$. By Lemma 9 , the voltages assigned to the co-tree $\operatorname{arcs}(i, \beta)$, where $1 \leqslant i \leqslant p-1$, must be distinct non-identity elements of $\mathbb{Z}_{p}$. Up to relabeling the vertices, i.e., up to an automorphism of $\Gamma=K_{2, p}$, there is a unique way to assign voltages to the edges. By Lemma 7, there is at most one such cover of $K_{2, p}$ up to isomorphism of covering projections.

It only remains to show that this covering projection has the desired properties. Let $H=\langle\xi\rangle$, viewed additively, and without a loss of generality assume that $\xi_{i \beta}=i \xi$ for all $i$. Note that the closed walks based at the vertex $\alpha$ are generated by the closed walks of the form $(\alpha, i, \beta, 0, \alpha), 1 \leqslant i \leqslant p-1$. Thus by Lemma 8 an automorphism $g$ of $\Gamma=K_{2, p}$ lifts to $\tilde{g}$ if and only if there exists a solution to the equations $\xi_{i \beta}^{g^{\#}}=\xi_{W_{i}^{g}}$ for all $i$, where $W_{i}=(\alpha, i, \beta, 0, \alpha)$ and $g^{\#} \in \operatorname{Aut}(H)$. 
Given a primitive element $t$ of the field $\operatorname{GF}(p)$, let $g$ be the $(p-1)$-cycle $\left(1 t t^{2} \ldots t^{p-2}\right)$ in $\operatorname{Sym}\left(\Delta_{2}\right)$, and assume that $g$ fixes both $\alpha$ and $\beta$. Then, for all $i>0$, assuming that $i \equiv t^{k_{i}}(\bmod p), \xi_{i \beta}^{g^{\#}}=\xi_{t^{k_{i} \beta}}^{g^{\#}}=\left(t^{k_{i}} \xi\right)^{g^{\#}}$; on the other hand, $\xi_{W_{i}^{g}}=\xi_{\left(t^{k_{i}}\right)^{g} \beta}=\left(t^{k_{i}}\right)^{g} \xi=$ $t^{k_{i}+1} \xi$. Indeed, these equations are satisfied by the outer automorphism $\nu$ of $H$ given by $\nu: \xi \mapsto t \xi$, and so the $(p-1)$-cycle $g$ lifts.

Let $h$ be the $p$-cycle $\left(\begin{array}{lll}0 & 1 \ldots(p-1))\end{array}\right)$ in $\operatorname{Sym}\left(\Delta_{2}\right)$. Then $\xi_{i \beta}^{h^{\#}}=(i \xi)^{h^{\#}}$; on the other hand, $\xi_{W_{i}^{h}}=-\xi_{1 \beta}+\xi_{(i+1) \beta}=-\xi+(i+1) \xi=i \xi$. These equations are satisfied by the trivial automorphism of $H$, and so $h$ lifts. The group generated by $h$ and $g$ acts 2-transitively on $\Delta_{2}$ while leaving all of $\Delta_{1}$ fixed.

Next, let $x$ be the automorphism of $K_{2, p}$ that interchanges $\alpha$ and $\beta$ but fixes all other vertices. We will now associate the voltage $\xi_{i \beta}$ with the closed walk $U_{i}:=(0, \alpha, i, \beta, 0)$ based at 0 . In order for $x$ to lift, there must exist $x^{\#} \in \operatorname{Aut}(H)$ such that $\xi_{i \beta}^{x \#}=\xi_{U_{i}^{x}}=\xi_{i \beta}^{-1}$ for all $i \in \Delta_{2} \backslash\{0\}$. This corresponds to the outer automorphism $\zeta: \xi \mapsto-\xi$, and so $x$ lifts as well. The group generated by $x$ acts 2 -transitively on $\Delta_{1}$ while leaving all of $\Delta_{2}$ fixed. It is easy to see that the group $G=\langle g, h, x\rangle$ lifts and $\Gamma$ is locally $(G, 3)$-arc-transitive. By [5, Theorem 3], this unique cover (up to isomorphism of covers) will be a locally 3 -arc-transitive graph.

Finally, we suppose that $m=n=p$, where $p$ is an odd prime. By Lemma $17, H \cong \mathbb{Z}_{p}$. By Lemma 9, the voltages assigned to the edges incident with a single vertex are distinct and all come from $\mathbb{Z}_{p}$. Up to relabeling the vertices, i.e., up to an automorphism of $K_{p, p}$, there is a unique way to do this. Namely, if we let $\Delta_{1}=\left\{\alpha_{0}, \alpha_{1}, \ldots, \alpha_{p-1}\right\}$, let $\Delta_{2}=\{0,1, \ldots, p-1\}$, and assume that the spanning tree with identity voltages consists of all edges incident with either $\alpha_{0}$ or 0 , we will assign voltages to co-tree edges by letting $\xi_{i \alpha_{j}}$ be the $(i, j)$-entry of the following $(p-1) \times(p-1)$ matrix:

$$
\left(\begin{array}{ccclc}
\xi & 2 \xi & 3 \xi & \ldots & (p-1) \xi \\
2 \xi & 2(2 \xi) & 3(2 \xi) & \ldots & (p-1)(2 \xi) \\
3 \xi & 2(3 \xi) & 3(3 \xi) & \ldots & (p-1)(3 \xi) \\
\vdots & \vdots & \vdots & & \vdots \\
(p-1) \xi & 2((p-1) \xi) & 3((p-1) \xi) & \ldots & (p-1)((p-1) \xi)
\end{array}\right)
$$

It is clear that each row and each column of the matrix contains each nonidentity element of $\langle\xi\rangle$ exactly once. Hence we have a voltage assignment which satisfies all the above lemmas, and, up to permuting the vertices of $K_{p, p}$, this assignment is unique. Arguing again as in the $K_{2, p}$ case, we see that the desired automorphisms lift, and so we again get a cover of the desired type that is unique up to isomorphism of covering projections.

\section{Locally 3-arc-transitive regular covers of complete bipartite graphs with elementary abelian covering group}

In this section, we will assume that the group of covering transformations is elementary abelian; that is, we will assume that $H:=\mathrm{CT}(\mathcal{P}) \cong \mathbb{Z}_{p}^{d}$, where $p$ is a prime and $d \geqslant 2$ 
(the case $d=1$ falls under the cyclic case). We begin by proving Theorem 3 , which is essentially a corollary of Theorem 1 :

Proof of Theorem 3. In this case, $H_{\beta} \cong \mathbb{Z}_{p}^{f}$ for some $f \leqslant d$. Thus $\operatorname{Aut}\left(H_{\beta}\right) \cong \mathrm{GL}_{f}(p) \lesssim$ $\mathrm{GL}_{d}(p)$, and the result follows.

By Theorem 1, the process of finding the locally 3 -arc-transitive covers of $K_{m, n}$ comes down to finding locally 3 -arc-transitive covers of $K_{2, m}$ and $K_{2, n}$. While it can be extremely difficult to determine which $K_{2, m}$ have lifts in full generality, there are certain cases that are not so difficult.

Proposition 19. Up to isomorphism of covering projections, $\Gamma=K_{2, p^{f}}$ has a unique locally 3-arc-transitive regular cover $\tilde{\Gamma}$ with covering projection $\mathcal{P}: \tilde{\Gamma} \rightarrow \Gamma$ such that $H=\mathrm{CT}(\mathcal{P}) \cong \mathbb{Z}_{p}^{f}$.

Proof. By Lemma 9, a different nonidentity element of $H$ must be assigned as a voltage to each co-tree edge of $\Gamma$. Up to relabeling the vertices, i.e., up to an automorphism of $\Gamma$, there is a unique way to do this, and so, by Lemma 7 , there is at most one such cover.

Let the biparts of $\Gamma$ be $\Delta_{1}=\{\alpha, \beta\}$ and $\Delta_{2}=\left\{0,1, \ldots, p^{f}-1\right\}$. Define $W_{i}$ to be the closed walk $(\alpha, i, \beta, 0, \alpha), 1 \leqslant i \leqslant p^{f}-1$. The $W_{i}$ form a generating set for all closed walks based at $\alpha$. We will show that this particular voltage assignment results in a locally 3 -arc-transitive regular cover $\tilde{\Gamma}$. First, let $h \in \operatorname{Aut}(\Gamma)$ be the automorphism that fixes $\Delta_{2}$ but interchanges $\alpha$ and $\beta$. Thus

$$
\xi_{W_{i}^{h}} \leftrightarrow(\alpha, i, \beta, 0, \alpha)^{h}=(\beta, i, \alpha, 0, \beta) \leftrightarrow-\xi_{i \beta}=-\xi_{W_{i}} .
$$

Since there is always an automorphism inverting all elements of an abelian group, by Lemma $8, h$ lifts.

We will view $H$ additively as a vector space, and we label the elements of $H$ as $\overrightarrow{0}, \ldots, \overrightarrow{\left(p^{f}-1\right)}$, where $\vec{i}$ is the voltage of the arc $(i, \beta)$. The $W_{i}$ generate a set of closed walks based at $\alpha$, and the voltage of $W_{i}$ is $\vec{i}$. Suppose $g \in \operatorname{Aut}(\Gamma)$ such that $g$ fixes $\alpha$, $\beta$, and 0 . Then

$$
\xi_{W_{i}^{g}} \leftrightarrow(\alpha, i, \beta, 0, \alpha)^{g}=\left(\alpha, i^{g}, \beta, 0, \alpha\right) \leftrightarrow \overrightarrow{i^{g}} .
$$

Thus any $g \in \operatorname{Aut}(\Gamma)$ that acts on $\left\{1, \ldots, p^{f}-1\right\}$ as an element of $\mathrm{GL}_{f}(p)$ acts on $\left\{\overrightarrow{1}, \ldots, \overrightarrow{p^{f}-1}\right\}$ will lift to an automorphism of $\tilde{\Gamma}$.

Now, let $g_{i}, 0 \leqslant i \leqslant p^{f}-1$, be defined to be the permutation of $\left\{0, \ldots, p^{f}-1\right\}$ such that $0^{g_{i}}=i$ and $j^{g_{i}}=k$, where $\xi_{k \beta}$ has voltage corresponding to $\vec{i}+\vec{j}$. Since the voltages on edges incident with $\beta$ are all distinct, this is well-defined permutation. Hence

$$
\xi_{W_{j}^{g_{i}}} \leftrightarrow(\alpha, j, \beta, 0, \alpha)^{g_{i}}=(\alpha, k, \beta, i, \alpha) \leftrightarrow-\vec{i}+\vec{i}+\vec{j}=\vec{j}=\xi_{W_{j}} .
$$

Since $\xi_{W_{j}}^{g_{i}^{\#}}=\xi_{W_{j}}$ is satisfied for all $j$ by letting $g_{i}^{\#}$ be the trivial automorphism of $H, g_{i}$ lifts. Therefore, a group of automorphisms $X$ of $\Gamma$ stabilizing each element in $\Delta_{1}$ that acts isomorphically to $\operatorname{AGL}_{f}(p)$ on $\Delta_{2}$ lifts. Since the group $G=\langle X, h\rangle$ lifts, and $\Gamma$ is locally $(G, 3)$-arc-transitive, $\tilde{\Gamma}$ is locally $(\tilde{G}, 3)$-arc-transitive, as desired. 
Corollary 20. Let $H \cong \mathbb{Z}_{p}^{d}$. The graph $K_{2, p^{f}}$ has a locally 3 -arc-transitive regular cover with $\mathrm{CT}(\mathcal{P}) \lesssim H$ if and only if $1 \leqslant f \leqslant d$.

Proof. If $f>d$, then there are not enough distinct elements of $H$ to assign as voltages to arcs of $K_{2, p^{f}}$, a contradiction. If $f \leqslant d$, then we choose a subgroup $K \leqslant H$ such that $K \cong \mathbb{Z}_{p}^{f}$. The result follows from Proposition 19 .

Combined with Theorem 3, Proposition 19 shows that finding the possible $m$ for which $K_{2, m}$ has a locally 3 -arc-transitive regular cover with elementary abelian covering transformations isomorphic to $\mathbb{Z}_{p}^{d}$ comes down to representation theory over $\operatorname{GF}(p)$. We will now use these results to classify the complete bipartite graphs with locally 3-arctransitive regular covers whose covering transformation group is a subgroup of $\mathbb{Z}_{p} \times \mathbb{Z}_{p}$, where $p$ is a prime.

Lemma 21. Let $p, r$ be distinct primes. If $\mathbb{Z}_{r}^{f} \lesssim G L_{d}(p)$, then $f \leqslant d$.

Proof. This is a basic exercise in representation theory and is left to the reader.

Proposition 22. Let $H \cong \mathbb{Z}_{p} \times \mathbb{Z}_{p}$, where $p$ is a prime. The only values of $m$ for which the complete bipartite graph $K_{2, m}$ has a locally 3-arc-transitive regular cover whose covering transformations are a subgroup of $H$ are $m=2,3, p$, and $p^{2}$.

Proof. Let the biparts of $\Gamma=K_{2, m}$ be $\Delta_{1}=\{\alpha, \beta\}$ and $\Delta_{2}$. Let $G \leqslant \operatorname{Aut}(\Gamma)$ be the subgroup of automorphisms that lifts to $\tilde{\Gamma}$ such that $\tilde{\Gamma}$ is locally $(\tilde{G}, 3)$-arc-transitive. By Theorem 3, if $S:=\operatorname{soc}\left(G_{\alpha \beta}^{\Delta_{2}}\right)$, then either $S \cong \mathbb{Z}_{p}^{f}$ for some $f \in \mathbb{N}$ or $G_{\alpha \beta}^{\Delta_{2}} \lesssim \mathrm{GL}_{2}(p)$. By Corollary 20, if $S \cong \mathbb{Z}_{p}^{f}$ then $f=1,2$, and both $K_{2, p}$ have $K_{2, p^{2}}$ have such covers, which are unique up to isomorphism of covering projections by Proposition 19. We also know that $K_{2,2}$ has such a cover by Theorem 2. By [21, Proposition 6.4], $K_{2,3}$ has a unique such cover (up to isomorphism of covering projections), and all of $\operatorname{Aut}\left(K_{2,3}\right)$ lifts.

Assume now that $G_{\alpha \beta}^{\Delta_{2}} \lesssim \mathrm{GL}_{2}(p)$ and $\left|\Delta_{2}\right|>3$. By [18, Proposition 5.5.10], no nonabelian finite simple group has a nontrivial representation of degree 2 in odd characteristic, and since $\left|\mathrm{GL}_{2}(2)\right|=6$, no nonabelian finite simple group is isomorphic to a subgroup of $\mathrm{GL}_{2}(2)$. Hence $S \cong Z_{r}^{f}$ for some prime $r \neq p$, and, by Lemma $21, f \leqslant 2$.

Suppose first that $f=2$. Let $G_{\alpha \beta} \cong X \leqslant \mathrm{GL}_{2}(p)$, and let $X=N K$, where $N \cong$ $\operatorname{soc}\left(G_{\alpha \beta}^{\Delta_{2}}\right)$ and $K \cong G_{\alpha \beta v}$, where $v \in \Delta_{2}$. Since $N$ is a minimal normal subgroup of $X$ and $G F(p)^{*}$ is cyclic, $N$ is in the kernel of the determinant map. Moreover, the center of $\mathrm{SL}_{2}(p)$ has order 2 , so either $N \cong \mathbb{Z}_{2}^{2}$ or $N \lesssim \operatorname{PSL}_{2}(p)$. The only subgroups of $\mathrm{PSL}_{2}(p)$ isomorphic to $\mathbb{Z}_{r}^{2}, r \neq p$, are isomorphic to $\mathbb{Z}_{2}^{2}[3]$. Hence $N \cong \mathbb{Z}_{2}^{2}$ and so $G_{\alpha \beta} \cong A_{4}$. However, $A_{4}$ has no faithful representation of degree 2, a contradiction. Hence $f=1$, and $G_{\alpha \beta} \cong \mathbb{Z}_{r}: \mathbb{Z}_{r-1} \cong \mathrm{AGL}_{1}(r)$, where $r>3$. However, when $r-1>2$, the group $\mathbb{Z}_{r}: \mathbb{Z}_{r-1}$ has no faithful representation of degree 2 (see, for instance, [17, Theorem 25.10]), a final contradiction.

Once the locally 3-arc-transitive regular covers of $K_{2, m}$ and $K_{2, n}$ have been found, it remains to be seen whether $K_{m, n}$ has a locally 3 -arc-transitive regular cover. In certain cases, we can give a definitive answer. 
Proposition 23. If $\Gamma=K_{p^{f}, p^{d}}$, where $f$ divides $d$, then there exists a regular cover $\tilde{\Gamma}$ and a covering projection $\mathcal{P}: \tilde{\Gamma} \rightarrow \Gamma$ such that $\tilde{\Gamma}$ is locally 3 -arc-transitive and $\mathrm{CT}(\mathcal{P}) \cong \mathbb{Z}_{p}^{d}$.

Proof. Let $V=\mathbb{Z}_{p}^{d}$ and let $\tau$ be a generator of $\mathrm{GL}_{1}\left(p^{d}\right) \leqslant \mathrm{GL}_{d}(p)$. Since $f$ divides $d$, $p^{f}-1$ divides $p^{d}-1$, and hence $\sigma=\tau^{\frac{p^{d}-1}{p^{f}-1}}$ has order $p^{f}-1$, stabilizes a decomposition of $V$ into $\left(p^{d}-1\right) /\left(p^{f}-1\right)$ subspaces of size $p^{f}-1$, and acts semiregularly on the non-zero elements of $V$.

Let the biparts of $\Gamma$ be $\Delta_{1}=\left\{\gamma_{0}, \ldots, \gamma_{p^{f}-1}\right\}$ and $\Delta_{2}=\left\{\delta_{0}, \ldots, \delta_{p^{d}-1}\right\}$. We choose a nonzero element $\xi \in V$, and to each $\operatorname{arc}\left(\delta_{i}, \gamma_{j}\right)$, where $1 \leqslant i \leqslant p^{d}-1$ and $1 \leqslant j \leqslant p^{f}-1$, we assign the voltage $\xi_{i j}=\xi_{\delta_{i} \gamma_{j}}=\xi^{\tau^{i} \sigma^{j}}=\xi^{\sigma^{j} \tau^{i}}$. Note that for any fixed $i$, the voltages $\xi_{i j}, \xi_{i j^{\prime}}$ are distinct for $j \neq j^{\prime}$, and, similarly, for any fixed $j$, the voltages $\xi_{i j}$ and $\xi_{i^{\prime} j}$ are distinct for $i \neq i^{\prime}$. We define further the closed walk $W_{i j}=\left(\gamma_{0}, \delta_{i}, \gamma_{j}, \delta_{0}, \gamma_{0}\right)$ based at $\gamma_{0}$, and define the closed walk $U_{i j}=\left(\delta_{0}, \gamma_{0}, \delta_{i}, \gamma_{j}, \delta_{0}\right)$ based at $\delta_{0}$.

We will show first that the element $g \in \operatorname{Aut}(\Gamma)$ lifts, where $g$ acts like the cycle $\left(\begin{array}{llll}\delta_{1} & \delta_{2} & \ldots & \delta_{p^{d}-1}\end{array}\right)$ on $\Delta_{2}$ and trivially on $\Delta_{1}$. In this case, we need to find $g^{\#} \in \operatorname{Aut}(V)$ that satisfies:

$$
\begin{aligned}
\left(\xi^{\tau^{i} \sigma^{j}}\right)^{g^{\#}} & =\xi_{i j}^{g^{\#}}=\xi_{W_{i j}^{g}} \leftrightarrow\left(\gamma_{0}, \delta_{i}, \gamma_{j}, \delta_{0}, \gamma_{0}\right)^{g} \\
& =\left(\gamma_{0}, \delta_{(i+1) \bmod \left(p^{d}-1\right)}, \gamma_{j}, \delta_{0}, \gamma_{0}\right) \leftrightarrow \xi^{\tau^{i+1} \sigma^{j}}=\left(\xi^{\tau^{i} \sigma^{j}}\right)^{\tau},
\end{aligned}
$$

and $g^{\#}=\tau$ demonstrates that $g$ lifts.

We will show next that the element $h \in \operatorname{Aut}(\Gamma)$ lifts, where $h$ acts like the cycle $\left(\begin{array}{lllll}\gamma_{1} & \gamma_{2} & \ldots & \gamma_{p^{f}-1}\end{array}\right)$ on $\Delta_{1}$ and trivially on $\Delta_{2}$. In this case, we need to find $h^{\#} \in \operatorname{Aut}(V)$ that satisfies:

$$
\begin{aligned}
\left(\xi^{\tau^{i} \sigma^{j}}\right)^{h^{\#}} & =\xi_{i j}^{h^{\#}}=\xi_{W_{i j}^{h}} \leftrightarrow\left(\gamma_{0}, \delta_{i}, \gamma_{j}, \delta_{0}, \gamma_{0}\right)^{h} \\
& =\left(\gamma_{0}, \delta_{i}, \gamma_{(j+1) \bmod \left(p^{f}-1\right)}, \delta_{0}, \gamma_{0}\right) \leftrightarrow \xi^{\tau^{i} \sigma^{j+1}}=\left(\xi^{\tau^{i} \sigma^{j}}\right)^{\sigma},
\end{aligned}
$$

and $h^{\#}=\sigma$ demonstrates that $h$ lifts.

We will next show that a group $X$ acting regularly on $\Delta_{2}$ and trivially on $\Delta_{1}$ lifts. We will define the element $x_{k} \in X$ that sends $\delta_{0}^{x_{k}}$ to $\delta_{k}$. We then define $\delta_{i}^{x_{k}}=\delta_{\ell}$, where $\xi_{\ell j}=\xi_{i j}+\xi_{k j}$. In this case, we need $x_{k}^{\#} \in \operatorname{Aut}(V)$ that satisfies:

$$
\xi_{i j}^{x_{k}^{\#}}=\xi_{W_{i j}^{x_{k}}} \leftrightarrow\left(\gamma_{0}, \delta_{i}, \gamma_{j}, \delta_{0}, \gamma_{0}\right)^{x_{k}}=\left(\gamma_{0}, \delta_{\ell}, \gamma_{j}, \delta_{k}, \gamma_{0}\right) \leftrightarrow-\xi_{k j}+\xi_{\ell j}=\xi_{i j},
$$

and $x_{k}^{\#}=1 \in \operatorname{Aut}(V)$ satisfies these equations, and so $x_{k}$ lifts. A group $Y$ acting regularly on $\Delta_{1}$ and trivially on $\Delta_{2}$ is shown to lift analagously (the walks $U_{i j}$ are a generating set for the closed walks based at $\delta_{0}$ ). The group that lifts acts locally 3 -arc-transitively on $\Gamma$, and hence $\tilde{\Gamma}$ is locally 3 -arc-transitive as well.

Lemma 24. Let $p$ be a prime. If $\Gamma=K_{3, p^{2}}$, then there exists a regular cover $\tilde{\Gamma}$ of $\Gamma$ with covering projection $\mathcal{P}: \tilde{\Gamma} \rightarrow \Gamma$ such that $\tilde{\Gamma}$ is locally 3 -arc-transitive and $\mathrm{CT}(\mathcal{P}) \cong \mathbb{Z}_{p} \times \mathbb{Z}_{p}$. 
Proof. First, if $p=3$, then the result follows from Proposition 23. Let $\tau$ be a generator of $\mathrm{GL}_{1}\left(p^{2}\right)$. If $p \neq 3$, then $p^{2} \equiv 1(\bmod 3)$, and so we can find $\sigma=\tau^{\frac{p^{2}-1}{3}}$ of order 3 . Note also that since $\sigma \in\langle\tau\rangle,[\sigma, \tau]=1$. We define the biparts of $\Gamma$ to be $\Delta_{1}=\{\alpha, \beta, \gamma\}$ and $\Delta_{2}=\left\{0,1, \ldots, p^{2}-1\right\}$. If $V=\mathbb{Z}_{p} \times \mathbb{Z}_{p}$ and $\xi$ is a nonidentity element of $V$ such that $\xi^{\sigma} \notin\langle\xi\rangle$, we define the voltage on the arc $(i, \beta)$ to be $\xi_{i \beta}=\xi^{\tau^{i}}$ and the voltage on the arc $(i, \gamma)$ to be $\xi_{i \gamma}=\xi_{i \beta}+\xi_{i \beta}^{\sigma}=\xi^{\tau^{i}}+\xi^{\tau^{i} \sigma}$. We define $W_{i \epsilon}$ to be the closed walk $(\alpha, i, \epsilon, 0, \alpha)$ and $U_{i \epsilon}$ to be the closed walk $(0, \alpha, i, \epsilon, 0)$.

We will show first that the element $g \in \operatorname{Aut}(\Gamma)$ lifts, where $g$ acts like the cycle $\left(\begin{array}{lll}1 & 2 \ldots p^{n}-1\end{array}\right)$ on $\Delta_{2}$ and trivially on $\Delta_{1}$. In this case, we need to find $g^{\#} \in \operatorname{Aut}(V)$ that satisfies:

$$
\begin{aligned}
\left(\xi^{\tau^{i}}\right)^{g^{\#}} & =\xi_{i \beta}^{g^{\#}}=\xi_{W_{i \beta}^{g}} \leftrightarrow(\alpha, i, \beta, 0, \alpha)^{g} \\
& =\left(\alpha,(i+1) \bmod \left(p^{n}-1\right), \beta, 0, \alpha\right) \leftrightarrow \xi^{\tau^{i+1}}=\left(\xi^{\tau^{i}}\right)^{\tau}, \\
\left(\xi^{\tau^{i}}+\xi^{\tau^{i} \sigma}\right)^{g^{\#}}= & \xi_{i \gamma}^{g^{\#}}=\xi_{W_{i \gamma}^{g}} \leftrightarrow(\alpha, i, \gamma, 0, \alpha)^{g}=\left(\alpha,(i+1) \bmod \left(p^{n}-1\right), \gamma, 0, \alpha\right) \\
\leftrightarrow & \xi^{\tau^{(i+1)}}+\xi^{\tau^{i+1} \sigma}=\left(\xi^{\tau^{i}}+\xi^{\tau^{i} \sigma}\right)^{\tau},
\end{aligned}
$$

and hence $g^{\#}=\tau$ demonstrates that $g$ lifts.

We will show now that the element $x \in \operatorname{Aut}(\Gamma)$ lifts, where $x$ acts like the cycle $(\alpha \beta \gamma)$ on $\Delta_{1}$ and trivially on $\Delta_{2}$. In this case, we need to find $x^{\#} \in \operatorname{Aut}(V)$ that satisfies:

$$
\begin{gathered}
\left(\xi^{\tau^{i}}\right)^{x^{\#}}=\xi_{i \beta}^{x^{\#}}=\xi_{U_{i \beta}^{x}} \leftrightarrow(0, \alpha, i, \beta, 0)^{x}=(0, \beta, i, \gamma, 0) \leftrightarrow \xi_{i \gamma}-\xi_{i \beta}=\left(\xi^{\tau^{i}}\right)^{\sigma}, \\
\left(\xi^{\tau^{i}}+\xi^{\tau^{i} \sigma}\right)^{x^{\#}}=\xi_{i \gamma}^{x^{\#}}=\xi_{U_{i \gamma}^{x}} \leftrightarrow(0, \alpha, i, \gamma, 0)^{x}=(0, \beta, i, \alpha, 0) \leftrightarrow-\xi_{i \beta} .
\end{gathered}
$$

Since for any $\eta \in V,\left(\eta+\eta^{\sigma}+\eta^{\sigma^{2}}\right)^{\sigma}=\left(\eta+\eta^{\sigma}+\eta^{\sigma^{2}}\right)$, and $\sigma$ fixes only the identity $0 \in V$, for any $\eta \in V$ we have $-\eta=\eta^{\sigma}+\eta^{\sigma^{2}}$. Thus,

$$
\left(\xi^{\tau^{i}}+\xi^{\tau^{i} \sigma}\right)^{x^{\#}}=-\xi_{i \beta}=\left(\xi^{\tau^{i}}+\xi^{\tau^{i} \sigma}\right)^{\sigma}
$$

and hence $x^{\#}=\sigma$ demonstrates that $x$ lifts.

We will show now that a group $Y$ acting regularly on $\Delta_{2}$ and trivially on $\Delta_{1}$ lifts. We will define the element $y_{k} \in Y$ that sends $0^{y_{k}}$ to $k$. We then define $i^{y_{k}}=\ell$, where $\xi_{\ell \beta}=\xi_{i \beta}+\xi_{k \beta}$. In this case, we need $y_{k}^{\#} \in \operatorname{Aut}(V)$ that satisfies:

$$
\begin{gathered}
\xi_{i \beta}^{y_{k}^{\#}} \leftrightarrow(\alpha, i, \beta, 0, \alpha)^{y_{k}}=(\alpha, \ell, \beta, k, \alpha) \leftrightarrow-\xi_{k \beta}+\xi_{\ell \beta}=\xi_{i \beta}, \\
\xi_{i \gamma}^{y_{k}^{\#}} \leftrightarrow(\alpha, i, \gamma, 0, \alpha)^{y_{k}}=(\alpha, \ell, \gamma, k, \alpha) \leftrightarrow-\xi_{k \gamma}+\xi_{\ell \gamma}=\left(\xi_{\ell \beta}-\xi_{k \beta}\right)+\left(\xi_{\ell \beta}-\xi_{k \beta}\right)^{\sigma}=\xi_{i \gamma},
\end{gathered}
$$

and hence $y_{k}^{\#}=1 \in \operatorname{Aut}(V)$ demonstrates that $y_{k}$ lifts. 
Finally, we will show that an element $h$ lifts, where $h$ stabilizes $0, p^{2}-1, \alpha$ and satisfies $\beta^{h}=\gamma, \gamma^{h}=\beta$. We will define the action of $h$ on $\Delta_{2} \backslash\left\{0, p^{2}-1\right\}$ as follows. The elements $\xi, \xi^{\sigma}$ form a basis for the vector space $V$, and there is a linear transformation $\rho \in \operatorname{Aut}(V)$ such that $\xi^{\rho}=\xi+\xi^{\sigma}$ and $\left(\xi^{\sigma}\right)^{\rho}=-\xi^{\sigma}$. For any $i \in \Delta_{2} \backslash\left\{0, p^{2}-1\right\}$, we define $i^{h}=\ell$, where $\xi_{i \gamma}^{\rho}=\left(\xi^{\tau^{i}}+\xi^{\tau^{i} \sigma}\right)^{\rho}=\xi^{\tau^{\ell}}$. This is a well-defined automorphism of $\Gamma$, and to see that it lifts, we need to find $h^{\#} \in \operatorname{Aut}(V)$ such that:

$$
\begin{gathered}
\left(\xi^{\tau^{i}}\right)^{h^{\#}}=\xi_{i \beta}^{h^{\#}}=\xi_{W_{i \beta}^{h}} \leftrightarrow(\alpha, i, \beta, 0, \alpha)^{h}=(\alpha, \ell, \gamma, 0, \alpha) \leftrightarrow \xi_{\ell \gamma}=\xi^{\tau^{\ell}}+\xi^{\tau^{\ell} \sigma} \\
\left(\xi^{\tau^{i}}+\xi^{\tau^{i} \sigma}\right)^{h^{\#}}=\xi_{i \gamma}^{h^{\#}}=\xi_{W_{i \gamma}^{h}} \leftrightarrow(\alpha, i, \gamma, 0, \alpha)^{h}=(\alpha, \ell, \beta, 0, \alpha) \leftrightarrow \xi_{\ell \beta}=\left(\xi^{\tau^{i}}+\xi^{\tau^{i} \sigma}\right)^{\rho} .
\end{gathered}
$$

Now, since the elements $\xi, \xi^{\sigma}$ form a basis, there exist $a_{i}, b_{i} \in \mathbb{Z}_{p}$ such that $\xi^{\tau^{i}}=a_{i} \xi+b_{i} \xi^{\sigma}$. Hence,

$$
\begin{aligned}
\xi^{\tau^{i}}+\xi^{\tau^{i} \sigma} & =a_{i} \xi+b_{i} \xi^{\sigma}+a_{i} \xi^{\sigma}+b_{i} \xi^{\sigma^{2}} \\
& =a_{i} \xi+b_{i} \xi^{\sigma}+a_{i} \xi^{\sigma}+b_{i}\left(-\xi-\xi^{\sigma}\right) \\
& =\left(a_{i}-b_{i}\right) \xi+a_{i} \xi^{\sigma}
\end{aligned}
$$

This means that

$$
\xi^{\tau^{\ell}}=\left(\xi^{\tau^{i}}+\xi^{\tau^{i} \sigma}\right)^{\rho}=\left(\left(a_{i}-b_{i}\right) \xi+a_{i} \xi^{\sigma}\right)^{\rho}=\left(a_{i}-b_{i}\right) \xi-b_{i} \xi^{\sigma},
$$

and a similar calculation shows that

$$
\left(\xi^{\tau^{i}}\right)^{\rho}=a_{i} \xi+\left(a_{i}-b_{i}\right) \xi^{\sigma}=\xi^{\tau^{\ell}}+\xi^{\tau^{\ell} \sigma}
$$

Therefore, $h^{\#}=\rho$ demonstrates that $h$ lifts. The group generated by these automorphisms both lifts and acts locally 3 -arc-transitively on $\Gamma$, and therefore $\tilde{\Gamma}$ is a locally 3 -arctransitive regular cover of $\Gamma$.

Lemma 25. Let $p$ be a prime. If $\Gamma=K_{3, p}$, then there exists a regular cover $\tilde{\Gamma}$ of $\Gamma$ with covering projection $\mathcal{P}: \tilde{\Gamma} \rightarrow \Gamma$ such that $\tilde{\Gamma}$ is locally 3 -arc-transitive and $\mathrm{CT}(\mathcal{P}) \cong \mathbb{Z}_{p} \times \mathbb{Z}_{p}$.

Proof. First, if $p=2$ or $p=3$, then the result follows from [21, Proposition 6.4] or Theorem 2, respectively. Let $V=\mathbb{Z}_{p} \times \mathbb{Z}_{p}$, and let $\xi, \eta$ be two elements of $V$ such that $\langle\xi\rangle \cap\langle\eta\rangle=0$, the identity element of $V$, and let $\Gamma=K_{3, p}$ have biparts $\Delta_{1}=\{\alpha, \beta, \gamma\}$ and $\Delta_{2}=\{0,1, \ldots, p-1\}$. For each $i \in \Delta_{2} \backslash\{0\}$ (which is naturally identified with $\mathbb{Z}_{p} \backslash\{0\}$ ), we assign to the $\operatorname{arc}(i, \beta)$ the voltage $\xi_{i \beta}=i \xi$, and we assign to the arc $(i, \gamma)$ the voltage $\xi_{i \gamma}=i \eta$. A group stabilizing $\Delta_{1}$ and acting like $\operatorname{AGL}_{1}(p)$ on $\Delta_{2}$ lifts by a proof analogous to that of Theorem 2. The automorphism $\sigma \in \operatorname{Aut}(V)$ defined by $\xi^{\sigma}=\eta-\xi$ and $\eta^{\sigma}=-\xi$ demonstrates that the automorphism $x \in \operatorname{Aut}(\Gamma)$ that acts trivially on $\Delta_{2}$ and as the 3-cycle $(\alpha \beta \gamma)$ on $\Delta_{1}$ lifts, and the automorphism $\rho \in \operatorname{Aut}(V)$ defined by $\xi^{\rho}=\eta$ and $\eta^{\rho}=\xi$ demonstrates that the automorphism $h \in \operatorname{Aut}(\Gamma)$ that acts trivially on $\Delta_{2}$ and as the transposition $(\beta \gamma)$ on $\Delta_{1}$ lifts. Therefore, the cover $\tilde{\Gamma}$ derived from this voltage assignment is locally 3 -arc-transitive, as desired. 
In general, it is not guaranteed that $K_{m, n}$ has a locally 3 -arc-transitive regular cover even if $K_{2, m}$ and $K_{2, n}$ both have locally 3 -arc-transitive regular covers with covering transformation groups isomorphic to a subgroup of $\mathbb{Z}_{p}^{d}$, as the following lemma shows:

Lemma 26 ([20, Proposition 3.3]). Let $p \neq 3$ be a prime. Then $K_{3,3}$ does not have a locally 3-arc-transitive regular cover with covering transformation group $Z_{p} \times Z_{p}$.

We can now prove Theorem 4 .

Proof of Theorem 4. First, the result follows for $K_{2,2}, K_{2,3}, K_{2, p}, K_{2, p^{2}}, K_{3, p}, K_{3, p^{2}}, K_{p, p}$, $K_{p, p^{2}}$, and $K_{p^{2}, p^{2}}$ by Theorem 2, Propositions 22 and 23, and Lemmas 24 and 25. The only other possibility is $K_{3,3}$, which is ruled out by Lemma 26 .

Proof of Corollary 5. By Theorem 4, we only must consider $K_{2,2}, K_{2,3}, K_{2, p}, K_{2, p^{2}}, K_{3, p}$, $K_{3, p^{2}}, K_{p, p}, K_{p, p^{2}}$, and $K_{p^{2}, p^{2}}$. However, $K_{2,2}$ is just $C_{4}$, which has only one co-tree edge in a spanning tree, and can be eliminated immediately. For $K_{2,3}$, the uniqueness follows from [21, Proposition 6.4], and for the other graphs the uniqueness follows from Proposition 12 and reasoning as in the proof of Theorem 2 .

It should be noted that all of the covers obtained in Theorems 2 and 4 have induced local 2-transitive actions that are affine groups. On the other hand, if $E$ is the edge set of a graph $\Gamma$ and $T$ is the edge set of a spanning tree of $\Gamma$, [21, Proposition 6.4] shows that assigning the elements of a basis of $\mathbb{Z}_{p}^{|E \backslash T|}$ to the co-tree edges of $\Gamma$ induces a regular cover such that the full automorphism group $\operatorname{Aut}(\Gamma)$ lifts. On the other hand, a group $G$ that has a 2-transitive action on $m$ elements and a degree $d$ representation over $\operatorname{GF}(p)$ does not actually guarantee that a voltage assignment from $\mathbb{Z}_{p}^{d}$ to the arcs of $K_{2, m}$ exists such that $G$ lifts.

Example 27. Even though $A_{5} \lesssim \mathrm{GL}(3,11)$, there is no voltage assignment from $Z_{11}^{3}$ to $K_{2,5}$ such that $A_{5}$ lifts.

To see this, we can use the algorithm described in [21]. Indeed, the dual representation of $A_{5}$ on $H_{1}\left(K_{2,5} ; \mathbb{Z}_{11}\right) \cong \mathbb{Z}_{11}^{4}$ leaves no 3 -dimensional space invariant, and hence no such cover can exist.

The cases when $d>2$ can be approached similarly. Theorem 3 reduces the problem of finding the possible values of $m$ for which $K_{2, m}$ can have a locally 3 -arc-transitive cover whose covering transformation group is isomorphic to a subgroup of $\mathbb{Z}_{p}^{d}$ to which 2 -transitive groups can have a $d$-dimensional representation over $G F(p)$. From there, if $K_{2, m}$ and $K_{2, n}$ both have locally 3 -arc-transitive covers with covering transformation group isomorphic to a subgroup of $\mathbb{Z}_{p}^{d}$, then the algorithm presented in [21] can be used to determine whether or not $K_{m, n}$ has a locally 3 -arc-transitive cover with covering transformation group isomorphic to a subgroup of $\mathbb{Z}_{p}^{d}$. 


\section{Acknoledgements}

The author acknowledges the support of the Australian Research Council Discovery Grant DP120101336 during his time spent at The University of Western Australia and would like to thank the anonymous referee, whose comments and critiques greatly improved the content and presentation from a previous version.

\section{References}

[1] M.D.E. Conder and J. Ma. Arc-transitive abelian regular covers of cubic graphs. J. Algebra 387:215-242, 2013.

[2] A. Delgado and B. Stellmacher. Weak (B,N)-pairs of rank 2. In Groups and Graphs: New Results and Methods, pages 58-244. Birkhäuser Verlag, 1985.

[3] L.E. Dickson. Linear groups: With an exposition of the Galois field theory. with an introduction by W. Magnus. Dover Publications Inc., New York, 1958.

[4] J. Dixon and B. Mortimer. Permutation Groups. Springer, 1996.

[5] D. Ž. Djoković. Automorphisms of graphs and coverings. J. Combinatorial Theory Ser. B, 16:243-247, 1974.

[6] S.-F. Du, J.H. Kwak, and M.-Y. Xu. 2-arc-transitive regular covers of complete graphs having the covering transformation group $Z_{p}^{3}$. J. Combin. Theory Ser. B, 93:73-93, 2005.

[7] S.-F. Du, D. Marušič, and A.O. Waller. On 2-arc-transitive covers of complete graphs. J. Combin. Theory Ser. B, 74:276-290, 1998.

[8] S.F. Du, J.H. Kwak, and M.Y. Xu. Lifting of automorphisms on the elementary abelian regular coverings. Lin. Alg. Appl., 373:101-119, 2003.

[9] W. Fan, D. Leemans, C.H. Li, and J. Pan. Locally 2-arc-transitive complete bipartite graphs. J. Combin. Theory Ser. A, 120:683-699, 2013.

[10] X.G. Fang, C. H. Li, and C.E. Praeger. The locally 2-arc transitive graphs admitting a Ree simple group. J. Algebra, 282:638-666, 2004.

[11] M. Giudici, C.H. Li, and C.E. Praeger. Analysing finite locally $s$-arc transitive graphs. Trans. Amer. Math. Soc., 356(1):291-317, 2004.

[12] M. Giudici, C.H. Li, and C.E. Praeger. Characterizing finite locally $s$-arc transitive graphs with a star normal quotient. J. Group Theory, 9:641-658, 2006.

[13] M. Giudici, C.H. Li, and C.E. Praeger. Locally s-arc transitive graphs with two different quasiprimitive actions. J. Algebra, 299:863-890, 2006.

[14] D. Gorenstein, R. Lyons, and R. Solomon. The Classification of Finite Simple Groups, volume 40 of A.M.S. Math. Surveys and Monographs. AMS, 1994.

[15] J.L. Gross and T.W. Tucker. Topological Graph Theory. Dover, 2001.

[16] A.A. Ivanov, R.A. Liebler, T. Penttila, and C.E. Praeger. Antipodal distancetransitive covers of complete bipartite graphs. Europ. J. Combin., 18:11-33, 1997. 
[17] G. James and M. Liebeck. Representations and Characters of Groups. Cambridge University Press, 1993.

[18] P. Kleidman and M. Liebeck. The subgroup structure of the finite classical groups. London Mathematical Society Lecture Note Series, 129, 1990.

[19] D. Leemans. Locally $s$-arc transitive graphs related to sporadic simple groups. $J$. Algebra, 322:882-892, 2009.

[20] Aleksander Malnič, Dragan Marušič, and Primož Potočnik. On cubic graphs admitting an edge-transitive solvable group. J. Algebraic Combin., 20(1):99-113, 2004.

[21] A. Malnič, D. Marušič, and P. Potočnik. Elementary abelian covers of graphs. J. Alg. Combin., 20:71-97, 2004.

[22] A. Malnič, R. Nedela, and M. S̆koviera. Lifting graph automorphisms by voltage assignments. Europ. J. Combin., 21:927-947, 2000.

[23] C. E. Praeger. An O'Nan-Scott theorem for finite quasiprimitive permutation groups and an application to 2-arc transitive graphs. J. London Math. Soc., 47:227-239, 1993.

[24] C.E. Praeger. Finite quasiprimitive graphs. In Surveys in combinatorics, 1997. Proceedings of the 16th British combinatorial conference, volume 241 of London Math. Soc. Lecture Note Ser., pages 65-85. Cambridge University Press, July 1997.

[25] E. Swartz. The locally 2-arc transitive graphs admitting an almost simple group of Suzuki type. J. Combin. Theory Ser. A, 119:949-976, 2012.

[26] J. Tits. Moufang octagons and the Ree groups of type ${ }^{2} F_{4}$. Amer. J. Math., 105:539$594,1983$.

[27] W.T. Tutte. A family of cubical graphs. Proc. Cambridge Philos. Soc., 43:459-472, 1947.

[28] W.T. Tutte. On the symmetry of cubic graphs. Canad. J. Math., 11:621-624, 1959.

[29] J. van Bon and B. Stellmacher. Locally s-transitive graphs. J. Algebra, 441:243-293, 2015.

[30] R. Weiss. The nonexistence of 8-transitive graphs. Combinatorica, 1:309-311, 1981.

[31] R. Weiss. Generalized polygons and s-transitive graphs. In Finite geometries, buildings, and related topics, pages 95-103. Oxford Univ. Press, 1990. 